Canadian Journal of Medicine

\title{
Meta-Analysis of Prevalence of CFTR \\ Mutations in Middle East Populations
}

\section{Antimicrobial Resistance among Urinary Tract Isolates of Patients Suffering from Urinary Tract Infections (UTIs): A Retrospective Observational Study}

\section{Mohammadreza Ferasatpour, Mohammadkarim Rahimi, Atieh Asadollah*}

Faculty of medicine, Islamic Azad University of Medical Science, Tehran, Iran

Keywords:

UTI, Enterococcus, Drug resistance

\section{Received}

21 March 2020

Received in revised form 03 April 2020

Accepted

18 April 2020

\begin{abstract}
The current study was performed to determine the frequency and drug resistance and biotypes of enterococcus-related urinary tract infections (UTI) in a center in Tehran. In this observational cross-sectional descriptive study, 2235 consecutive patients with suspected UTI were enrolled and evaluated for frequency, drug resistance, and biotypes of enterococcusrelated urinary tract infections. Our findings revealed that 2589 subjects $(6.5 \%)$ had established UTI among which 87 subjects (3.4\%) exhibited enterococcus-related urinary tract infection. Among them 70 cases were evaluated for biotype and drug resistance that all cases were Faecalis biotype. The nitrofurantoin $(6 \%)$ and gentamicin $(85.9 \%)$ were found to be the lowest and most drug resistance, respectively.,Ceftizoxime, ciprofloxacine, co-trimoxazole ampicillin, and nalidixic acid were among the least active agents for the UTI isolates of $E$. coli Morovere, a divers rang of antibiotic resistance has been shown for other antibiotics in . .the present studyUTI isolates of klebsiella showed resistance to ampicillin, followed by staphylococcus aureus (penicillin and tetracycline), staphylococcus epidermis isolates (penicillin), staphylococcus saprophyticus (gentamycin, penicillin, and cloxacillin), and streptococcus viridans ciprofloxacin (44\%) and tetracycline (83\%). Our findings revealed that $E$ coli is the most common cause of urinary tract infection in both men and women. Enterococcus is responsible for three percent of urinary tract infections with dominant biotype of faecalis. The most sensitivity and resistance were related to nitrofurantoin and gentamicin, respectively.
\end{abstract}




\section{Introduction}

Urinary tract infections are the most common bacterial infections in children and older people, resulting in clinic or emergency visits or hospital admissions and subsequent morbidity and mortality, where the loss of appropriate treatment can have serious consequences [1-3]. Nowadays, appropriate therapeutic strategy for UTIs is considered as a challenge because of high resistance to commonly used antibiotics word wide, especially in Gram-negative organisms [4-7]. The availability of new information about bacteria sensitivity and the familiarity with the common resistance among the local bacteria can be helpful in therapeutic management.

By assessing the antimicrobial susceptibility pattern, an increasing amount of antibiotic resistance associated with urinary tract infections such as cephazolin, gentamicin and ampicillin has been determined. Thus, the choice of appropriate agent and surveillance of antibiotic resistance patterns could be of great importance for empirical treatment, leading to prevention of drug resistance and recommendations of first choice antibiotic therapy need to be based on practical rationales including cultivation, particularly in complicated UTIs, antibiotic susceptibility data and promoting appropriate use of antibiotics [8]. While misuse of antibiotics has been suggested to be associated with antibiotic resistance through the mutant strains, and unresolved, relapsed UTIs [910]. Current study aimed to assess antibiotic resistance patterns and biotypes of enterococcusrelated UTI, and to address challenges linked to this important issue.

\section{Method}

A total of 2235 people referred to a specialized center of Dr shariati hospital were included in the study. Our syudy popolation conssited of 1390 (62\%) women and 842 (38\%) men. Among 301 infected patients enrolled in the study, $224(74 \%)$ were women and 77(26\%) were men. Cultivation and antibiogram test were performed according to CLSI standard [11]. Data in relation to antimicrobials, and microbiological findings were also recorded for exploring the impact of antimicrobial resistance.

\section{Results}

As shown in Table 1, the number of the infected men and women in terms of the type of bacteria and their rate. The most common infection in women belonged to Escherichia coli, followed by Staphylococcus, streptococcus viridians, klebsiella, staphylococcus epidermis, Enterococcus, Proteus, pseudomonas, and saprophyticus, while Escherichia coli, enterococcus, streptococcus viridans, Klebsiella, Proteus, pseudomonas, staphylococcus aureus, staphylococcus epidermis, staphylococcus saprophticus were among the most infection in men. Table 1 shows that, Escherichia coli is the most important pathogen from UTIs. Furthrmore, UTIs are more common in women than men.

The results of antibiogram has been indicated in tables $2,3,4,5,6$, and 7 according to the type of bacteria. Based on the results presented herein, E.coli was found to be highly sensetive to nitrofurantoin, and gentamycin, while many agents including ceftizoxime, ciprofloxacine, co,trimoxazole .ampicillin, nalidixic acid were among the least active agents

According to the Table 3, Klebsiella was sensitive ceftriaxone, gentamicin, ciprofloxacin, and ceftizoxime, while resistance to ampicillin among UTI isolates of klebsiella was also revealed. As shown in Table 4, among staphylococcus aureus isolates collected from UTI, resistant to penicillin and tetracycline were observed. in contrast, the use of vancomycin and nitrofurantoin were all highly active (100\% and 94\%). Among staphylococcus epidermis isolates, nitrofurantoin, gentamycin, vancomycin, and cloxacillin are considerably active $(\geq 80 \%)$, except for penicillin with $8 \%$ sensitivity (Table 5). Nitrofurantoin and Vancomycin were found to be the most active agents with $0 \%$ of the UTI isolates of staphylococcus saprophyticus being resistant, while gentamycin, 
penicillin, and cloxacillin were identified to be the least active agents with $20 \%$ of the isolates being resistant (Table 6). Additionally, among the UTI isolates of streptococcus viridans vancomycin $(100 \%)$ was one of the most active agents, followed by nitrofurantoin $(91 \%)$ penicillin $(74 \%)$, and ampicillin (70\%), while ciprofloxacin (56\%) and tetracycline (17\%) was among the least active agents.

Table 1

The Number of the Infected Based on the Type of Bacteria

\begin{tabular}{lll}
\hline Bacteria & Women & Men \\
\hline E.coli & $149(66 \%)$ & $45(58 \%)$ \\
klebsiella & $18(\% 8)$ & $4(\% 5)$ \\
aureus & $13(\% 5)$ & $4(\% 5)$ \\
Enterococcus & $8(\% 3)$ & $9(\% 11)$ \\
Proteus & $3(\% 1)$ & $4(\% 5)$ \\
pseudomonas & $3(\% 1)$ & $3(\% 3)$ \\
viridians & $15(\% 6)$ & $6(\% 7)$ \\
epidermis & $12(\% 5)$ & $2(\% 2)$ \\
saprophyticus & $3(\% 1)$ & $0(\% 0)$ \\
& & 77 \\
\hline
\end{tabular}

Table 2

The Results of Antibiogram Based on the E.coli

\begin{tabular}{lccc}
\hline Anti-biotic & Sensitive & Semi-sensitive & Refractory \\
\hline Ciprofloxacin & $\% 60$ & $\% 39$ & $1 \%$ \\
Cefotaxime & $\% 67$ & $\% 2$ & $\% 31$ \\
Co-Trimoxazole & $\% 29$ & $\% 1$ & $\% 70$ \\
Nitrofurantoin & $\% 95$ & -1 & $\% 11$ \\
Ceftizoxime & $\% 89$ & $\% 1$ & $\% 28$ \\
Gentamycin & $\% 71$ & $\% 5$ & $\% 72$ \\
Ampicillin & $\% 23$ & $\% 3$ & $\% 32$ \\
Ceftriaxone & $\% 65$ & $\% 2$ & $\% 73$ \\
Nalidixic Acid & $\% 25$ & & $\% 1$ \\
\hline
\end{tabular}

Table 3

The Results of Antibiogram based on the Klebsiella

\begin{tabular}{lccc}
\hline Anti-biotic & Sensitive & Semi-sensitive & Refractory \\
\hline Ciprofloxacin & $\% 84$ & $\% 5$ & $\% 11$ \\
Ceftizoxime & $\% 89$ & - & $\% 31$ \\
Co-Trimoxazole & $\% 69$ & - & $\% 35$ \\
Nitrofurantoin & $\% 35$ & $\% 30$ & $\% 10$ \\
Gentamycin & $\% 85$ & - & $\% 100$ \\
Ampicillin & - & - & - \\
Ceftriaxone & $\% 100$ & - & $\% 37$ \\
Nalidixic Acid & $\% 63$ & 05 & $\%$ \\
\hline
\end{tabular}


Table 4

The Results of Antibiogram based on the Staphylococcus Aureus

\begin{tabular}{lccc}
\hline Anti-biotic & Sensitive & Semi-sensitive & Refractory \\
\hline Ciprofloxacin & $\% 45$ & $\% 11$ & $\% 57$ \\
Gentamycin & $\% 43$ & - & $\% 100$ \\
Penicillin & - & - & - \\
Vancomycin & $\% 100$ & - & $\% 64$ \\
Cloxacillin & $\% 36$ & - & $\% 6$ \\
Nitrofurantoin & $\% 94$ & - & $\% 100$ \\
Tetracycline & - & - & $\% 72$ \\
Co-Trimoxazole & $\% 28$ & - & \\
\hline
\end{tabular}

Table 5

The Results of Antibiogram based on the Staphylococcus Epidermis

\begin{tabular}{|c|c|c|c|}
\hline Anti-biotic & Sensitive & Semi-sensitive & Refractory \\
\hline Nitrofurantoin & $\% 85$ & - & $\% 15$ \\
\hline Gentamycin & $\% 80$ & - & $\% 20$ \\
\hline Penicillin & $\% 8$ & - & $\% 92$ \\
\hline Vancomycin & $\% 100$ & - & - \\
\hline Cloxacillin & $\% 82$ & - & $\% 18$ \\
\hline
\end{tabular}

Table 6

The Results of Antibiogram based on the Staphylococcus Saprophyticus

\begin{tabular}{lccc}
\hline Anti-biotic & Sensitive & Semi-sensitive & Refractory \\
\hline Nitrofurantoin & $\% 100$ & - & $\% 80$ \\
Gentamycin & $\% 20$ & - & $\% 80$ \\
Penicillin & $\% 20$ & - & - \\
Vancomycin & $\% 100$ & - & $\% 80$ \\
Cloxacillin & $\% 20$ & - & - \\
\hline
\end{tabular}

Table 7

The Results of Antibiogram based on the Streptococcus Viridans

\begin{tabular}{lccc}
\hline Anti-biotic & Sensitive & Semi-sensitive & Refractory \\
\hline Ciprofloxacin & $\% 56$ & - & $\% 44$ \\
Ampicillin & $\% 70$ & - & $\% 30$ \\
Penicillin & $\% 74$ & - & - \\
Vancomycin & $\% 100$ & $\% 46$ & $\% 79$ \\
Tetracycline & $\% 17$ & - & $\% 9$ \\
Nitrofurantoin & $\% 91$ & - & $\%$ \\
\hline
\end{tabular}




\section{Discussion}

Our findings revealed that $E$ coli is the most common cause of urinary tract infection in both men and women. Nitrofurantoin, and gentamycin were found to bethe most active agents for the UTI isolates of $E$. coli, while ceftizoxime, ciprofloxacine, co-trimoxazole ampicillin, and nalidixic acid were among the least active agents Furtmore, a divers rang of antibiotic resistance has been shown . for other antibiotics in the present studyUTI isolates of klebsiella showed resistance to ampicillin, followed by staphylococcus aureus (penicillin and tetracycline), staphylococcus epidermis isolates (penicillin), staphylococcus saprophyticus (gentamycin, penicillin, and cloxacillin), and streptococcus viridans ciprofloxacin (44\%) and tetracycline (83\%).

This resistance revealed in these antibiotics suggest that considerable caution should be taken in to consideration when choosing to use of broad-spectrum antibiotics as their widespread use may lead to resistance. van Driel et al. [8] reported that E. coli was the most frequently detected uropathogen (83\%), followed by Klebsiella pneumonia (5\%) and Klebsiella oxytoca (1.5\%). Aforementioned study indicated that the susceptibility of E. coli ranged from $66 \%$ for amoxicillin to $94 \%$ for ciprofloxacin and $100 \%$ for both nitrofurantoin and fosfomycin. Another study indicated increased pattern of resistance rate to co-amoxiclav along with amoxicillin in urinary pathogens among women in a UK hospital [12].

Escherichia coli has been reported as the most UTI isolates (76\%). Furthermore, enterobacteriaceae isolates were found previously to be considerably sensitive to amoxicillinclavulanate, nitrofurantoin, and fosfomycin, while $>20 \%$ were recorded as resistant to ciprofloxacin and co-trimoxazole (Ho et al, 2019). Additionally, E. coli represented as the most species in urine culture confirmed UTI of 538 women and exhibited resistance to the amoxicillin and trimethoprim/sulfamethoxazole [13].

The increase in resistance to many antibiotics makes the treatment of UTIs a challenging issue for managing patients. While nitrofurantoin is an active agent against E. coli but it is found to be less active against Klebsiella as a therapeutic option for uncomplicated UTIs. Rising antimicrobial resistance are linked to many reasons including overuse and over-the-counter use, prescription without indications (inappropriate or suboptimal), and unregulated use in livestock [14].

The development of new agent for the treatment of UTIs is not currently on the priority, where no effort has been dedicated, therefore, rising resistance and multi-drug-resistant pathogens lead to limited weapons for fighting the threat in complicated UTIs. judicious use of antibiotics is highly required in this circumstance under consideration of health care system [15]. Form the point of view for treatment, local levels of antimicrobial resistance may be capable of providing a better decision making for appropriate prescription choice in UTIs. Prospective, comparative investigations are required to address this issue.

\section{Conflict of interest}

None

\section{References}

[1] Mattoo TK, Asmar BI. Annotations on emerging concerns about antibiotic-resistant urinary tract infection. Pediatrics. 2020 Feb 1;145(2):e20193512

[2] Detweiler K, Mayers D, Fletcher SG. Bacteruria and urinary tract infections in the elderly. Urologic Clinics. 2015 Nov 1;42(4):561-8.

[3] Mouton CP, Bazaldua O V, Pierce B, Espino DV. Common infections in older adults. American Family Physician. 2001 Jan $15 ; 63(2): 257-68$.

[4] Bryce A, Hay AD, Lane IF, Thornton HV, Wootton M, Costelloe C. Global prevalence of antibiotic resistance in paediatric urinary tract infections caused by Escherichia coli and association with routine use of antibiotics in primary care: systematic review and meta-analysis. BMJ. 2016 Mar 15;352:1939. 
[5] Lukac PJ, Bonomo RA, Logan LK. Extended-spectrum $\beta$-lactamase-producing Enterobacteriaceae in children: old foe, emerging threat. Clinical Infectious Diseases. 2015 May 1;60(9):1389-97.

[6] Ho HJ, Tan MX, Chen MI, Tan TY, Koo SH, Koong AY, Ng LP, Hu PL, Tan KT, Moey PK, Koh EY. Interaction between antibiotic resistance, resistance genes, and treatment response for urinary tract infections in primary care. Journal of clinical microbiology. 2019 Sep 1;57(9):e00143-19.

[7] Frazee BW, Trivedi T, Montgomery M, Petrovic DF, Yamaji R, Riley L. Emergency Department Urinary Tract Infections Caused by Extended-Spectrum $\beta$-Lactamase-Producing Enterobacteriaceae: Many Patients Have No Identifiable Risk Factor and Discordant Empiric Therapy Is Common. Ann Emerg Med. 2018;72(4):449-56.

[8] van Driel AA, Notermans DW, Meima A, Mulder M, Donker GA, Stobberingh EE, Verbon A. Antibiotic resistance of Escherichia coli isolated from uncomplicated UTI in general practice patients over a 10-year period. European Journal of Clinical Microbiology \& Infectious Diseases. 2019 Nov 1;38(11):2151-8.

[9] Baquero F. Low-level antibacterial resistance: a gateway to clinical resistance. Drug Resistance Updates. 2001 Apr 1;4(2):93105.

[10] Kim HY, Lee SJ, Lee DS, Yoo JM, Choe HS. Microbiological characteristics of unresolved acute uncomplicated cystitis. Microbial Drug Resistance. 2016 Jul 1;22(5):387-91.

[11] Clinical and Laboratory Standards Institute. (2005) Performance standards for antimicrobial susceptibility testing: fifteenth informational supplement M100-S15. CLSI, Wayne. M100, 29th ed. January 2019 Replaces M100, 28th ed.

[12] Baines G, Banjoko A, Brair A, Gray J, Desai N, Cardozo L, Toozs-Hobson P. Antibiotic resistance in urinary tract infections: A re-visit after five years and experience over two sites. Post Reproductive Health. 2020 Apr 6:2053369120910039.

[13] Rossignol L, Vaux S, Maugat S, Blake A, Barlier R, Heym B, Le Strat Y, Blanchon T, Hanslik T, Coignard B. Incidence of urinary tract infections and antibiotic resistance in the outpatient setting: a cross-sectional study. Infection. 2017 Feb $1 ; 45(1): 33-40$.

[14] Ventola CL. The antibiotic resistance crisis: part 1: causes and threats. Pharmacy and Therapeutics. 2015 Apr;40(4):277-83.

[15] Paul R. State of the globe: Rising antimicrobial resistance of pathogens in urinary tract infection. J Glob Infect Dis. 2018 JulSep;10(3):117-8. 